Pacific Journal of Mathematics

AMENABLE GROUPS FOR WHICH EVERY TOPOLOGICAL 


\title{
AMENABLE GROUPS FOR WHICH EVERY TOPOLOGICAL LEFT INVARIANT MEAN IS INVARIANT
}

\author{
Alan L. T. Paterson
}

Let $G$ be an amenable locally compact group. It is conjectured that every topological left invariant mean on $L_{\infty}(G)$ is (topologically) invariant if and only if $G \in[F C]^{-}$. This conjecture is shown to be true when $G$ is discrete and when $G$ is compactly generated.

1. Introduction. Let $G$ be an amenable locally compact group and let $\mathfrak{R}_{t}(G)\left(\Re_{t}(G)\right)$ be the set of topological left (right) invariant means on $L_{\infty}(G)$. A natural question to ask is: when does $\mathfrak{Q}_{t}(G)=$ $\Re_{t}(G)$ ? Obviously, $\mathfrak{R}_{t}(G)=\Re_{t}(G)$ if $G$ is compact or abelian. The results of this paper strongly support the conjecture that $\Omega_{t}(G)=$ $\Re_{t}(G)$ if and only if $G \in[F C]^{-}$, the class of those locally compact groups each of whose conjugacy classes is relatively compact. Theorem 3.2 (Theorem 4.4) establishes this conjecture when $G$ is discrete (compactly generated).

The present writer's interest in the above question arose from his inability to prove [1, Theorem 7]. The latter result asserts that if $G$ is an exponentially bounded discrete group, then $\mathfrak{R}_{t}(G)=\Re_{t}(G)$. This result is false. (See (3.3).)

I am indebted to Dr F. W. Ponting for help in translating portions of [1].

2. Preliminaries. The cardinality of a set $A$ is denoted $|A|$. Let $G$ be a group. The identity of $G$ will be denoted by $e$, and if $x \in G$, then $C_{x}=\left\{y x y^{-1}: y \in G\right\}$ is the conjugacy class of $x$ in $G$. If $a, x \in G$, then

$$
C(x)=\{y \in G: x y=y x\}, \quad C_{a}(x)=\left\{y \in G: y x y^{-1}=a\right\} .
$$

Now let $G$ be a locally compact group. The family of compact subsets of $G$ is denoted by $\mathscr{C}(G)$ and the family of compact neighborhoods of $e$ in $G$ is denoted by $\mathscr{C}_{e}(G)$. The algebra of continuous, bounded, complex-valued functions on $G$ is denoted by $C(G)$. Throughout the paper, $\lambda$ will be a left Haar measure on $G$. The group $G$ is called an $[F C]^{-}$group if $C_{x}$ is relatively compact for all $x \in G$. The class of discrete $[F C]^{-}$groups is denoted by $[F C]$. The group $G$ is called an $[I N]$ group if there exists $D \in \mathscr{C}_{e}(G)$ such that $x D=$ $D x$ for all $x \in G$. (For information about the classes $[F C]^{-}$and $[I N]$, 
see [4].)

Let $G$ be a locally compact group. For $\phi \in L_{\infty}(G)\left(=L_{1}(G)^{*}\right)$ and $\mu \in L_{1}(G)$, define $\phi \mu, \mu \phi \in L_{\infty}(G)$ by setting

$$
\phi \mu(\nu)=\phi(\mu * \nu), \quad \mu \phi(\nu)=\phi(\nu * \mu) \quad\left(\nu \in L_{1}(G)\right) .
$$

Let $P(G)$ be the set of probability measures in $L_{1}(G)$. A mean $M$ on $L_{\infty}(G)$ is said to be a topological left (right) invariant mean if

$$
M(\phi \mu)=M(\phi) \quad(M(\mu \phi)=M(\phi))
$$

for all $\phi \in L_{\infty}(G)$ and all $\mu \in P(G)$. The set of topological left (right) invariant means on $G$ is denoted by $\mathfrak{R}_{t}(G)\left(\Re_{t}(G)\right)$. A mean $M$ on $L_{\infty}(G)$ is said to be a topological invariant mean if $M \in \mathfrak{R}_{t}(G) \cap \Re_{t}(G)$. The group $G$ is amenable if and only if $\mathfrak{R}_{t}(G)\left(\Re_{t}(G)\right)$ is not empty. If $G$ is discrete, then $\mathfrak{R}_{t}(G)\left(\Re_{t}(G)\right)$ coincides with $\mathscr{L}(G)(\Re(G))$, the set of left (right) invariant means on $\iota_{\infty}(G)$. It is a simple consequence of the structure theory of $[F C]^{-}$groups that every $[F C]^{-}$group is amenable ([7], [5], [6]).

A measurable subset $T$ of $G$ is said to be topologically left (right) thick if

$$
\sup _{x \in C} \lambda(C \cap T x)=\lambda(C) \quad\left(\sup _{x \in C} \lambda(C \cap x T)=\lambda(C)\right)
$$

for all $C \in \mathscr{C}(G)$. The subset $T$ is topologically left (right) thick if and only if there exists $M \in \mathfrak{R}_{t}(G)\left(M \in \mathfrak{R}_{t}(G)\right)$ such that $M\left(\chi_{T}\right)=1$. (See [2, Theorem 7.8] and [12].) If $G$ is discrete, then $T$ is topologically left thick if and only if, for every finite subset $F$ of $G$, there exists $x_{F} \in G$ such that $F x_{F} \subset T$. In this case, $T$ is said to be left thick ([10]).

\section{The discrete case.}

LEMMA 3.1. Let $G$ be an amenable discrete group which is not an $[F C]$ group. Then $\mathfrak{L}(G) \neq \Re(G)$.

Proof. The result will follow once we have constructed a left thick subset $T$ of $G$ which is not right thick: for then any left invariant mean $M$ on $G$ for which $M\left(\chi_{T}\right)=1$ will not be right invariant.

To this end, let $\alpha$ be the smallest ordinal of cardinality $|G|$, and let $\left\{F_{\beta}: \beta \in \alpha\right\}$ be an enumeration of the family of finite subsets of $G$. Since $G \notin[F C]$, we can find $z \in G$ such that $C_{z}$ is infinite. Choose $z_{1}, z_{2}$ in $G$ such that $z_{1}^{-1} z_{2}=z$. The lemma will be proved once we have constructed (by transfinite recursion) a subset $\left\{x_{\beta}: \beta \in \alpha\right\}$ of $G$ such that for all $x \in G$ and all $\beta \in \alpha$, 


$$
x\left\{z_{1}, z_{2}\right\} \not \subset \cup\left\{F_{\delta} x_{\delta}: \delta \in \beta\right\} .
$$

(For then we can take $T=\cup\left\{F_{\beta} x_{\beta}: \beta \in \alpha\right\}$.) Suppose that $\beta \in \alpha$, and that elements $x_{\delta}(\delta \in \beta)$ have been constructed so that

$$
x\left\{z_{1}, z_{2}\right\} \not \subset \cup\left\{F_{\gamma} x_{\gamma}: \gamma \in \delta\right\}
$$

for all $x \in G$ and for all $\delta \in \beta$. Let $C=\cup\left\{F_{\delta} x_{\delta}: \delta \in \beta\right\}$. Note that $x\left\{z_{1}, z_{2}\right\} \not \subset C$ for all $x \in G$.

Let $y \in G$ and suppose that there exists $x \in G$ such that

$$
x\left\{z_{1}, z_{2}\right\} \subset C \cup F_{\beta} y .
$$

Then either $x z_{1} \in C, x z_{2} \in F_{\beta} y$ or $x z_{2} \in C, x z_{1} \in F_{\beta} y$ or $x z_{1} \in F_{\beta} y, x z_{2} \in F_{\beta} y$. If $x z_{1} \in C$ and $x z_{2} \in F_{\beta} y$, then $z=\left(x z_{1}\right)^{-1}\left(x z_{2}\right) \in C^{-} F_{\beta} y$. Applying a similar argument to each of the other cases, we see that either $z \in C^{-1} F_{\beta} y$ or $z^{-1} \in C^{-1} F_{\beta} y$ or $z \in y^{-1} F_{\beta}^{-1} F_{\beta} y$. Let $A=F_{\beta}^{-1} C z \cup F_{\beta}^{-1} C z^{-1}$. Note that $|A|<|G|$. Let $B=\left\{u \in G: u z u^{-1} \in F_{\beta}^{-1} F_{\beta}\right\}$. Then $y \in A \cup B$. We now show that $|G \sim B|=|G|$. It is elementary that if $a \in G$ and if $x_{a} \in G$ is such that $x_{a} z x_{a}^{-1}=a$, then $C_{a}(z)=x_{\alpha} C(z)$. If follows that $\left|C_{a}(z)\right|=$ $|C(z)|$ for all $a \in C_{z}$. If $|C(z)|=|G|$ and if $a \in C_{z} \sim F_{\beta}^{-1} F_{\beta}$, then $|G \sim B| \geqq\left|C_{a}(z)\right|=|G|$, and so $|G \sim B|=|G|$. If, on the other hand, $|C(z)|<|G|$, then $|B| \leqq\left|F_{\beta}^{-1} F_{\beta}\right||C(z)|<|G|$, and again $|G \sim B|=|G|$.

Since $|A|<|G|$ and $|G \sim B|=|G|$, we can find $x_{\beta} \in G \sim(A \cup B)$. As $A \cup B$ is the set of elements $y$ for which there exists $x$ satisfying (2), it follows that $x\left\{z_{1}, z_{2}\right\} \not \subset C \cup F_{\beta} x_{\beta}$ for all $x \in G$. This completes the construction of $\left\{x_{\beta}: \beta \in \alpha\right\}$ and hence the proof of the lemma.

THEOREM 3.2. Let $G$ be an amenable discrete group. Then $\mathfrak{L}(G)=\Re(G)$ if and only if $G \in[F C]$.

Proof. By (3.1), if $\mathcal{L}(G)=\Re(G)$, then $G \in[F C]$. Conversely, suppose that $G \in[F C]$. We could appeal to the result mentioned in (4.5), but the following easy proof is available.

Let $M \in \mathfrak{R}(G), x \in G$ and $E \subset G$. Since $C_{x}$ is finite, we can find $x_{1}, \cdots, x_{n}$ in $G$ such that $G$ is the disjoint union of the sets $x_{r} C(x)$. We can write $E=\bigcup_{r=1}^{n} x_{r} E_{r}$ where $E_{r} \subset C(x)$ for all $r$. Then

$$
M(E x)=\sum_{1}^{n} M\left(x_{r} E_{r} x\right)=\sum_{1}^{n} M\left(x_{r} x E_{r}\right)=\sum_{1}^{n} M\left(x_{r} E_{r}\right)=M(E),
$$

and $M \in \Re(G)$. It now follows that $\mathfrak{S}(G)=\Re(G)$.

Note 3.3. Contrary to the assertion of [1, Theorem 7], there are exponentially bounded groups $G$ for which $\mathfrak{L}(G) \neq \Re(G)$. An example of such a group is the (nilpotent) discrete group of upper triangular, real, $3 \times 3$ matrices with diagonal entries equal to 1 . (The latter 
group does not belong to $[F C]$.)

4. The nondiscrete case. We require three preliminary results.

Lemma 4.1. Let $G \in[I N]$ be such that for each $C \in \mathscr{C}(G)$, we have

$$
\sup _{D \in \mathscr{E}(G)}\left[\inf _{x \in G} \lambda\left(x C x^{-1} \cap D\right)\right]=\lambda(C) .
$$

Then the set $\cup\left\{x C x^{-1}: x \in G\right\}$ is relatively compact for each $C \in \mathscr{C}(G)$.

Proof. Let $U$ be an open, relatively compact subset of $G$. Approximating $U$ by compact subsets and using the equation (1), the fact that $G$ is unimodular, and the inner regularity of $\lambda$, we see that (1) is valid when $C$ is replaced by $U$.

The desired result will follow once it has been shown that there exists $D_{0} \in \mathscr{C}(G)$ such that $x U x^{-1} \subset D_{0}$ for all $x \in G$. Let $N$ be a compact, invariant neighborhood of $e$. Since $\bar{U}$ is compact, we can find $x_{1}, \cdots, x_{r}$ in $U$ such that

$$
U \subset \bigcup_{i=1}^{r} x_{i} N
$$

Then $k=\min _{i} \lambda\left(U \cap x_{i} N\right)$ is positive. Find $E \in \mathscr{C}(G)$ such that for all $x \in G$,

$$
\lambda\left(U \cap x^{-1} E x\right)=\lambda\left(x U x^{-1} \cap E\right)>\lambda(U)-k .
$$

Let $x_{0} \in G$. By (2) and (3), we can find, for each $i$, an element $n_{i} \in$ $N$ such that $x_{i} n_{i} \in x_{0}^{-1} E x_{0}$. So

$$
x_{i} N \subset x_{i} n_{i} N^{-1} N \subset x_{0}^{-1} E x_{0} N^{-1} N=x_{0}^{-1}\left(E N^{-1} N\right) x_{0},
$$

and it follows that $x_{0} U x_{0}^{-1} \subset E N^{-1} N$. Now take $D_{0}=E N^{-1} N$.

Lemma 4.2. Let $G$ be an amenable, compactly generated, locally compact group for which $\mathfrak{Q}_{t}(G)=\Re_{t}(G)$. Then $G \in[I N]$.

Proof. Assume that $\mathfrak{\Omega}_{t}(G)=\Re_{t}(G)$, and that $G$ is not an [IN] group. By [11, Theorem 1.8], we have

$$
\inf _{x \in G} \lambda\left(N \cap x^{-1} N x\right)=0
$$

for all $N \in \mathscr{C}_{e}(G)$. It easily follows that

$$
\inf _{x \in G} \lambda\left(N \cap x^{-1} M x\right)=0
$$

for all $N, M \in \mathscr{C}(G)$. 
Let $C \in \mathscr{C}_{e}(G)$ be such that $G=\bigcup_{n=1}^{\infty} C^{n}$, and let $\varepsilon=(1 / 2) \lambda(C)$. Using (1), we can find, for each $n$, an element $x_{n} \in G$ such that

$$
\lambda\left(C^{-1} C \cap x_{n}^{-1} C^{-n} C^{n} x_{n}\right)<\varepsilon 2^{-n} .
$$

Let $T=\bigcup_{n=1}^{\infty} C^{n} x_{n}$. It is obvious that $T$ is topologically left thick in $G$. The lemma will be established (by contradiction) once we have shown that $T$ is not topologically right thick.

Let $x \in G$, and, for each $n$, let $C_{n}=x C \cap C^{n} x_{n}$. Let $c_{n} \in C_{n}$. Then

$$
\lambda\left(C_{n}\right)=\lambda\left(c_{n}^{-1} C_{n}\right) \leqq \lambda\left(C^{-1} C \cap x_{n}^{-1} C^{-n} C^{n} x_{n}\right)<\varepsilon 2^{-n},
$$

using (2). It follows that $\lambda(x C \cap T)<\varepsilon \sum_{1}^{\infty} 2^{-n}=\varepsilon$, and so

$$
\lambda(x C \cap T) \leqq \frac{1}{2} \lambda(C) .
$$

So $T$ is not topologically right thick.

LEMMA 4.3. Let $G$ be an amenable, compactly generated, locally compact group for which $\mathfrak{L}_{t}(G)=\Re_{t}(G)$. Then

$$
\sup _{D \in \mathscr{C}(G)}\left[\inf _{x \in G} \lambda\left(x C x^{-1} \cap D\right)\right]=\lambda(C)
$$

for all $C \in \mathscr{C}(G)$.

Proof. Suppose that $C_{0} \in \mathscr{C}(G)$ is such that for some $\varepsilon>0$,

$$
\sup _{D \in \mathscr{E}(G)}\left[\inf _{x \in G} \lambda\left(x C_{0} x^{-1} \cap D\right)\right] \leqq \lambda\left(C_{0}\right)-\varepsilon .
$$

By (4.2), $G \in[I N]$, and hence is unimodular. It follows that (1) remains valid when $C_{0}$ is replaced by any larger compact subset of $G$. This fact will be used in the remainder of the proof.

Let $N$ be a compact, invariant neighborhood of $e$ and let $C \epsilon$ $\mathscr{C}(G)$ be such that $G=\bigcup_{n=1}^{\infty} C^{n}$ and $C_{0} \cup N \subset C$. We can suppose that $\lambda(N) \geqq \varepsilon$.

We now claim that if $D \in \mathscr{C}(G)$, and $\eta<\varepsilon$, then the set $A$, where

$$
A=\left\{x \in G: \lambda\left(x C x^{-1} \cap D\right) \leqq \lambda(C)-\eta\right\},
$$

is not relatively compact. For if $\bar{A} \in \mathscr{C}(G)$, and if $E=\bar{A} C(\bar{A})^{-1} \cup D$, then for all $x \in G$, we have $\lambda\left(x C x^{-1} \cap E\right) \geqq \lambda(C)-\eta>\lambda(C)-\varepsilon$, and the fact that (1) is valid, with $C_{0}$ replaced by $C$, is contradicted.

We now construct by induction a sequence $\left\{x_{n}\right\}$ in $G$ such that for each $x \in G$ and each positive integer $n$, we have

$$
\lambda\left(x C \cap\left(\bigcup_{r=1}^{n} C^{r} x_{r}\right)\right) \leqq\left(\lambda(C)-\frac{1}{2} \varepsilon\right) .
$$


Let $m$ be a positive integer and assume that $x_{1}, \cdots, x_{m-1}$ have been constructed such that (2) is valid for $1 \leqq n \leqq m-1$. Let $D=\bigcup_{r=1}^{m-1} C^{r} x_{r}$. Choose $x_{m}$ such that:

(i ) $x_{m} \notin C^{-m} D C^{-1} C$;

(ii) $\lambda\left(x_{m} C x_{m}^{-1} \cap N C^{-m} C^{m}\right) \leqq(\lambda(C)-(1 / 2) \varepsilon)$.

Let $x \in G$. We cannot have both of the sets $x C \cap D$ and $x C \cap C^{m} x_{m}$ not empty: for if this were so, then $D C^{-1} \cap C^{m} x_{m} C^{-1} \neq \varnothing$, and (i) is contradicted. So if $x C \cap D \neq \varnothing$, then (2) is trivially true with $n=m$.

Suppose then that $x C \cap D=\varnothing$, and set $E=x C \cap C^{m} x_{m}$. To complete the induction step, we show that

$$
\lambda(E) \leqq\left(\lambda(C)-\frac{1}{2} \varepsilon\right) .
$$

Two cases have to be considered. Suppose firstly that $x N \cap E=\varnothing$. Then

$$
\lambda(E) \leqq \lambda(x C \sim x N) \leqq \lambda(C)-\varepsilon<\left(\lambda(C)-\frac{1}{2} \varepsilon\right)
$$

and (3) is established. Now suppose that $x N \cap E \neq \varnothing$, and let $u \in N$ be such that $x u \in E$. Then

$$
(x u)^{-1} E \subset u^{-1} C \cap x_{m}^{-1} C^{-m} C^{m} x_{m},
$$

and since $N x_{m}^{-1}=x_{m}^{-1} N$, it follows that

$$
\lambda(E) \leqq \lambda\left(C \cap u x_{m}^{-1} C^{-m} C^{m} x_{m}\right) \leqq \lambda\left(x_{m} C x_{m}^{-1} \cap N C^{-m} C^{m}\right) .
$$

The inequality (3) now follows using (ii).

Now let $T=\bigcup_{n=1}^{\infty} C^{n} x_{n}$. The set $T$ is obviously topologically left thick in $G$. However, by $(2), \lambda(x C \cap T) \leqq \lambda(C)-1 / 2 \varepsilon$ for all $x \in G$, and so $T$ is not topologically right thick. It follows that $\ell_{t}(G) \neq$ $\Re_{t}(G)$, and the resultant contradiction establishes the lemma.

THEOREM 4.4. Let $G$ be an amenable, compactly generated, locally compact group. Then $\mathfrak{R}_{t}(G)=\Re_{t}(G)$ if and only if $G \in[F C]^{-}$.

Proof. Assume that $\mathfrak{R}_{t}(G)=\Re_{t}(G) . \quad$ By (4.3) and (4.1), we have $G \in[F C]^{-}$. Conversely, assume that $G \in[F C]^{-}$. Let $H$ be the closure of the commutator subgroup of $G$. By [4, Theorem 3.20], the group $H$ is compact. Let $\mu$ be the normalized Haar measure of $H$. In the obvious way, $\mu$ will be regarded as a probability measure on $G$. Note that if $M \in \mathfrak{R}_{t}(G)\left(\Re_{t}(G)\right)$ then $M(\phi \mu)=M(\phi)(M(\mu \phi)=M(\phi))$ for all $\phi \in L_{\infty}(G)$. Note also that $\delta_{h} * \mu=\mu=\mu * \delta_{h}$ for all $h \in H$.

Define 


$$
A=\{\phi \in C(G): \phi(x h)=\phi(x) \text { for all } x \in G \text { and all } h \in H\} .
$$

If $\phi \in A$ and $x, y \in G$, then, since $G / H$ is abelian, we have $x y=y x h_{0}$ for some $h_{0} \in H$, and it follows that $\phi(x y)=\phi(y x)$, and hence that $\nu \phi=\phi \nu$ for all $\nu \in P(G)$.

Now let $M \in \Re_{t}(G), \nu_{0}, \nu \in P(G)$ and $\psi \in L_{\infty}(G)$. Then if $x \in G$ and $h \in H$, we have

$$
\left(\mu \nu_{0}\right) \psi(x h)=\mu\left(\left.\left[\left(\nu_{0} \psi\right) x\right]\right|_{H} h\right)=\left(\mu \nu_{0}\right) \psi(x),
$$

and so $\left(\mu \nu_{0}\right) \psi \in A$. Now if $\nu \in P(G)$, we obtain

$$
M(\psi)=M\left(\nu\left(\mu \nu_{0}\right) \psi\right)=M\left(\left[\left(\mu \nu_{0}\right) \psi\right] \nu\right)=M(\psi \nu),
$$

and $M \in \mathfrak{R}_{t}(G)$. It easily follows that $\mathfrak{Q}_{t}(G)=\Re_{t}(G)$.

Note 4.5. The two theorems of this paper suggest the following conjecture: if $G$ is an amenable locally compact group, then $\mathfrak{R}_{t}(G)=$ $\Re_{t}(G)$ if and only if $G \in[F C]^{-}$. More evidence in support of this conjecture is found in the following result ([3], [8], [9]): if $G \in[S I N] \cap$ $[F C]^{-}$, then $\mathfrak{R}_{t}(G)=\Re_{t}(G)$.

\section{REFERENCES}

1. G. Adelson-Veskii and Ju. A. Šreider, The Banach mean on groups, Uspehi Mat. Nauk. (N.S.), 12 (1957), 131-136.

2. M. M. Day, Semigroups and amenability, Proceedings, Symposium on Semigroups, Wayne State University, Edited by K. Folley, Academic Press, 1969.

3. P. Gerl, Gleichverteilung auf lokalkompakten Gruppen, Math. Nachr., 71 (1976), 249-260.

4. S. Grosser and M. Moskowitz, Compactness conditions in topological groups, Jour. für die Reine und angewandte Math., 246 (1971), 1-40.

5. A. Hulanicki, On the spectral radius of hermitian elements in group algebras, Pacific J. Math., 18 (1966), 277-287.

6. - On positive functionals on a group algebra multiplicative on a subalgebra, Studia Math., 37 (1971), 163-171.

7. H. Leptin, Zur harmonischen Analyse klassenkompakter Gruppen, Invent. Math., 5 (1968), 249-254.

8. W. Maxones and H. Rindler, Asymptotisch gleichverteilte Massfolgen in Gruppen vom Heisenberg-Typ, Sitzungsber. Wien, Math. Nat. Kl IIa, 185 (1976), 485-504.

9. - Bemerkungen zu einer Arbeit von P. Gerl Gleichverteilung auf lokalkompakten Gruppen, Math. Nachr., 79 (1977), 193-199.

10. T. Mitchell, Constant functions and left invariant means on semigroups, Trans. Amer. Math. Soc., 119 (1965), 244-261.

11. W. Moran and J. H. Williamson, Isotone measures on groups, Math. Proc. Camb. Phil. Soc., 84 (1978), 89-107.

12. J. C.S. Wong, A characterization of topological left thick subsets in locally compact left amenable semigroups, Pacific J. Math., 62 (1976),295-303.

Received November 6, 1978.

UNIVERSITY OF ABERDEEN,

AberdeEn, Scotland 



\section{PACIFIC JOURNAL OF MATHEMATICS}

\section{EDITORS}

DONALD BABBITT (Managing Editor)

University of California

Los Angeles, CA 90024

HUGO RossI

University of Utah

Salt Lake City, UT 84112

C. C. Moore and ANDrew OGG

University of California

Berkeley, CA 94720
J. DUGUNDJI

Department of Mathematics University of Southern California Los Angeles, CA 90007

R. FINN and J. MILGRAM

Stanford University

Stanford, CA 94305

\section{ASSOCIATE EDITORS}
E. F. BeCKENBACH
B. H. NeumanN
F. WOLF
K. YOSHIDA

\section{SUPPORTING INSTITUTIONS}

UNIVERSITY OF BRITISH COLUMBIA CALIFORNIA INSTITUTE OF TECHNOLOGY UNIVERSITY OF CALIFORNIA MONTANA STATE UNIVERSITY UNIVERSITY OF NEVADA, RENO NEW MEXICO STATE UNIVERSITY OREGON STATE UNIVERSITY UNIVERSITY OF OREGON

\author{
UNIVERSITY OF SOUTHERN CALIFORNIA \\ STANFORD UNIVERSITY \\ UNIVERSITY OF HAWAII \\ UNIVERSITY OF TOKYO \\ UNIVERSITY OF UTAH \\ WASHINGTON STATE UNIVERSITY \\ UNIVERSITY OF · WASHINGTON
}

The Supporting Institutions listed above contribute to the cost of publication of this Journal, but they are not owners or publishers and have no responsibility for its content or policies.

Mathematical papers intended for publication in the Pacific Journal of Mathematics should be in typed form or offset-reproduced, (not dittoed), double spaced with large margins. Please do not use built up fractions in the text of the manuscript. However, you may use them in the displayed equations. Underline Greek letters in red, German in green, and script in blue. The first paragraph or two must be capable of being used separately as a synopsis of the entire paper. Please propose a heading for the odd numbered pages of less than 35 characters. Manuscripts, in triplicate, may be sent to any one of the editors. Please classify according to the scheme of Math. Reviews, Index to Vol. 39. Supply name and address of author to whom proofs should be sent. All other communications should be addressed to the managing editor, or Elaine Barth, University of California, Los Angeles, California, 90024.

50 reprints to each author are provided free for each article, only if page charges have been substantially paid. Additional copies may be obtained at cost in multiples of 50 .

The Pacific Journal of Mathematics is issued monthly as of January 1966. Regular subscription rate: $\$ 84.00$ a year (6 Vols., 12 issues). Special rate: $\$ 42.00$ a year to individual members of supporting institutions.

Subscriptions, orders for numbers issued in the last three calendar years, and changes of address should be sent to Pacific Journal of Mathematics, P.O. Box 969, Carmel Valley, CA 93924, U.S.A. Older back numbers obtainable from Kraus Periodicals Co., Route 100, Millwood, NY 10546.

PUBLISHED BY PACIFIC JOURNAL OF MATHEMATICS, A NON-PROFIT CORPORATION

Printed at Kokusai Bunken Insatsusha (International Academic Printing Co., Ltd.). 8-8, 3-chome, Takadanobaba, Shinjuku-ku, Tokyo 160, Japan.

Copyright (C) 1979 by Pacific Journal of Mathematics Manufactured and first issued in Japan 


\section{Pacific Journal of Mathematics}

\section{Vol. 84, No. $2 \quad$ June, 1979}

Somesh Chandra Bagchi and Alladi Sitaram, Spherical mean periodic

functions on semisimple Lie groups ........................ 241

Billy Joe Ball, Quasicompactifications and shape theory............. 251

Maureen A. Bardwell, The o-primitive components of a regular ordered permutation group ................................ 261

Peter W. Bates and James R. Ward, Periodic solutions of higher order

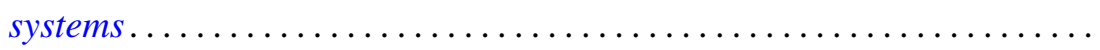

Jeroen Bruijning, A characterization of dimension of topological spaces by totally bounded pseudometrics......................... 283

Thomas Farmer, On the reduction of certain degenerate principal series

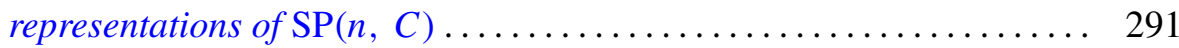

Richard P. Jerrard and Mark D. Meyerson, Homotopy with m-functions . . . . 305

James Edgar Keesling and Sibe Mardesic, A shape fibration with fibers of

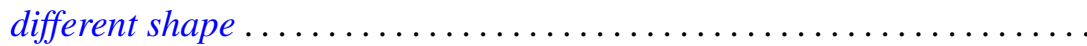

Guy Loupias, Cohomology over Banach crossed products. Application to bounded derivations and crossed homomorphisms ...............

Rainer Löwen, Symmetric planes ........................ 367

Alan L. T. Paterson, Amenable groups for which every topological left

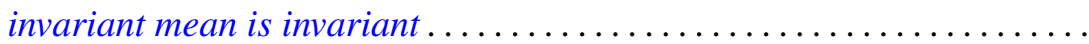

Jack Ray Porter and R. Grant Woods, Ultra-Hausdorff H-closed extensions

Calvin R. Putnam, Operators satisfying a $G_{1}$ condition .

Melvin Gordon Rothenberg and Jonathan David Sondow, Nonlinear smooth representations of compact Lie groups..............

Werner Rupp, Riesz-presentation of additive and $\sigma$-additive set-valued measures.

A. M. Russell, A commutative Banach algebra of functions of generalized variation

Judith D. Sally, Superregular sequences

Patrick Shanahan, On the signature of Grassmannians . . 